\title{
然
}

Agustín Navarro Vicente-Gella*

\section{LAS ALIANZAS PÚBLICO-PRIVADAS: VENTAJAS, INCONVENIENTES Y RIESGOS}

Tradicionalmente, los argumentos a favor de la provisión privada de servicios públicos han sido la mayor eficiencia y capacidad de innovación del sector privado, y la reducción de riesgos para la Administración cuando la responsabilidad de la provisión de un servicio pasa a recaer sobre una empresa privada. No obstante, los resultados empíricos de esta provisión privada no siempre han sido los esperados. Las alianzas público-privadas (PPP, por sus siglas en inglés) también presentan una serie de riesgos importantes, como se verá en este artículo, que deben ser abordados cuidadosamente, para lo cual la Oficina Nacional de Evaluación podría aportar un gran valor añadido si se convirtiera en una verdadera unidad de PPP de España.

Palabras clave: concesiones de obra o servicios, infraestructuras, reparto óptimo de riesgos, previsiones de demanda, responsabilidad patrimonial de la Administración, pasivo contingente, equidad intergeneracional.

Clasificación JEL: H54, L33, R42.

\section{Introducción}

Tradicionalmente, los argumentos más recurrentes a favor de la provisión privada de servicios públicos han sido la mayor eficiencia y capacidad de innovación del sector privado, y la reducción de riesgos para la Administración cuando la responsabilidad de la provisión de un servicio pasa a recaer sobre una empresa

\footnotetext{
* Técnico Comercial y Economista del Estado. Vocal asesor en la Dirección General de Política Económica.

El autor agradece a José Antonio Gómez-lbáñez sus lecciones sobre regulación y alianzas público-privadas en 2010 y las aportaciones realizadas en 2018 por Pedro Guerrero Meseguer, de las que se enriquece este artículo. No obstante, las opiniones expresadas en este documento son de exclusiva responsabilidad del autor.

Versión de octubre de 2019.

DOI: https:/doi.org/10.32796/bice.2019.3117.6931
}

privada. No obstante, las alianzas público-privadas no están exentas de cuestiones que es necesario abordar y resolver, como se verá en este artículo.

Aunque existen muchas modalidades de alianzas público-privadas (PPP por sus siglas en inglés de Estados Unidos [Department of Housing, Planning and Local Government -Ireland-, 2006]), la principal es la figura del contrato de concesión, que es, además, la principal figura de alianza público-privada que recoge el ordenamiento jurídico español. Por ello, este artículo se centrará en esta modalidad de alianza público-privada.

Muchos de los partidarios de la provisión privada de servicios de infraestructuras consideran que el principal problema que es $D$ 
necesario resolver es el compromiso con un conjunto de reglas justas y estables, y la principal preocupación es que la Administración pública pueda renegar posteriormente de sus compromisos (Gómez-lbáñez, 2006). Una vez que el concesionario ha construido la infraestructura concesionada, la Administración podría estar tentada de, por ejemplo, reducir las tarifas autorizadas, para beneficiar a los usuarios o a las cuentas públicas ${ }^{1}$, o incluso nacionalizar la concesión.

Este problema de oportunismo es general en los contratos que implican la realización de inversiones en activos específicos de la relación entre el proveedor y el cliente y se suelen resolver mediante la firma de un contrato de largo plazo entre las partes con carácter previo a la realización de las inversiones (Gómez-lbáñez, 2006).

En el caso de la provisión de servicios de infraestructuras, bajo este esquema, la Administración adjudica a una compañía privada una concesión o franquicia para la provisión de servicios específicos de infraestructuras por un periodo limitado de tiempo. La Administración representa, de hecho, a los usuarios al decidir la combinación de precios y calidad del servicio deseable. Con frecuencia, se adjudica el contrato de manera competitiva, frecuentemente al ofertante que ofrezca la menor tarifa para un nivel dado de servicio. También son frecuentes los contratos en los que la Administración fija tanto las prestaciones mínimas del servicio como las tarifas máximas, y el contrato se adjudica al participante que oferta el mayor canon o que solicita la menor subvención. Estos mecanismos competitivos dan cierta confianza a los consumidores de que los beneficios esperados por el concesionario no serán

1 En función de cuál sea el esquema de financiación de la concesión. excesivos respecto a los de las empresas que actúen en mercados competitivos. La Administración vigila el desempeño del concesionario para comprobar el cumplimiento del contrato, pero ninguna de las partes tiene potestad para modificar el contrato unilateralmente una vez que se ha adjudicado (Gómez-lbáñez, 2006).

\section{La experiencia en el resto del mundo}

Más allá del caso concreto de nuestro país, que luego analizaremos, los contratos de concesión fueron muy habituales en muchas zonas del mundo en el siglo xIx y fueron redescubiertos y mejorados a finales del siglo xx. En los años noventa, casi todas las nuevas vías de gran capacidad y muchas de las nuevas centrales eléctricas se construyeron por concesionarios privados. Al final del siglo, la mayoría de los principales ferrocarriles, servicios de suministro de agua y tratamiento de aguas residuales, compañías telefónicas y muchas de las compañías eléctricas de numerosos países, especialmente en Iberoamérica, habían sido privatizadas $u$ ofrecidas a concesionarios privados (Gómez-lbáñez, 2006).

No obstante, a principios de este siglo, algunos de los inversores privados no estaban satisfechos con la rentabilidad realmente obtenida en estos proyectos, mientras que los usuarios se mostraban en ocasiones escépticos respecto a que los niveles de tarifas y de servicios fueran los adecuados (Gómez-lbáñez, 2006).

Más allá del caso estricto de las concesiones, la experiencia del Reino Unido con los esquemas PPP, en general, también ha dado lugar a una profunda insatisfacción por una parte de la sociedad británica con estas modalidades $\triangleright$ 
de estructuración de los proyectos. Este fue uno de los países pioneros en el diseño de los PPP desde 1992. Según los defensores de estos proyectos, los PPP permitirían aumentar la inversión privada sin incrementar el endeudamiento del sector público y sin que los proyectos aparecieran en el balance del Estado, dando una impresión de gestión financiera prudente. En la mayoría de los casos, el pago al socio privado no procedía de los usuarios, sino de los presupuestos públicos. Por lo tanto, el sector privado adelantaba el coste de construcción del activo (colegios, hospitales, comisarías de Policía, etcétera) y el sector público se comprometía a realizar pagos anuales durante la vida del activo (Jubilee Debt Campaign, 2017).

Sin embargo, según algunos colectivos británicos, los resultados no han sido los esperados y, tras un aumento exponencial entre 1992 y 2007, desde entonces y hasta 2014 la utilización de esquemas PPP ha caído en el Reino Unido (Jubilee Debt Campaign, 2017).

\section{El caso español. Las concesiones de autopistas}

La experiencia en España con la modalidad contractual concesional se extiende a numerosos sectores y servicios públicos. Uno de los que presentan un historial más largo es el de las autopistas de peaje, por lo que nos centraremos en ellas.

Con sus numerosas modificaciones y derogaciones parciales, se sigue utilizando en la concesión de autopistas la Ley 8/1972, de 10 de mayo, de construcción, conservación y explotación de autopistas en régimen de concesión. Según el artículo primero de su versión en vigor, su objeto es «la regulación de las concesiones administrativas de construcción, conservación y explotación de autopistas y de las concesiones administrativas para la conservación y explotación de tramos de autopistas ya construidos». Según su artículo tercero, el «servicio objeto de la concesión constituye una actividad propia del Estado que el concesionario gestiona, en su nombre y temporalmente, bajo la inspección y vigilancia de la Administración concedente».

Desde un punto de vista más general, la normativa aplicable se enmarca en la Ley 9/2017, de 8 de noviembre, de Contratos del Sector Público, por la que se transponen al ordenamiento jurídico español las Directivas del Parlamento Europeo y del Consejo 2014/23/UE y 2014/24/UE, de 26 de febrero de 2014. Dicha ley contempla dos modalidades de alianzas público-privadas: el contrato de concesión de obras (artículo 14) y el contrato de concesión de servicios (artículo 15).

Según el artículo 14 de la ley, el «derecho de explotación de las obras (...) deberá implicar la transferencia al concesionario de un riesgo operacional en la explotación de dichas obras abarcando el riesgo de demanda o el de suministro, o ambos (...). La parte de los riesgos transferidos al concesionario debe suponer una exposición real a las incertidumbres del mercado que implique que cualquier pérdida potencial estimada en que incurra el concesionario no es meramente nominal o desdeñable». El artículo 15 también recalca que el «derecho de explotación de los servicios implicará la transferencia al concesionario del riesgo operacional».

Con nuestro marco jurídico se ha desarrollado un amplio número de concesiones en nuestro país. Por ejemplo, volviendo al caso de las autopistas de peaje, tras las primeras concesionadas en los años sesenta y setenta, a partir de finales de los noventa resurgió esta $\square$ 
modalidad contractual y se adjudicaron además las primeras concesiones de autopistas con peaje en sombra (Cañas, Sánchez, Vassallo y Castroman, 2006) y las primeras en la modalidad de pago por disponibilidad. Gracias a ello, en España existían 3.307 kilómetros de autopistas de peaje en régimen de concesión en 2017, todos abiertos al tráfico, cuya titularidad la ostentaban treinta sociedades concesionarias (Delegación del Gobierno en las Sociedades Concesionarias de Autopistas Nacionales de Peaje, 2018).

Tradicionalmente, la manera en que el ordenamiento jurídico español ha respondido a la necesidad de dar confianza a los inversores privados frente a la tentación de la Administración de nacionalizar la concesión, ha sido mediante la regulación de la Responsabilidad Patrimonial de la Administración (RPA). La legislación española ha regulado que, en caso de rescisión anticipada de la concesión, se tenía que abonar al concesionario el importe de las inversiones realizadas por razón de la expropiación de terrenos, ejecución de obras de construcción y adquisición de bienes que sean necesarios para la explotación de la obra objeto de la concesión, atendiendo a su grado de amortización (valor en libros del negocio).

El artículo 280 de la actual Ley de Contratos española sigue definiendo en los mismos términos la indemnización a la que tiene derecho el concesionario en caso de rescisión imputable a la Administración. En cambio, ha innovado con respecto a la ley anterior, al estipular que, cuando la causa de la rescisión no sea imputable a la Administración, ésta relicitará el contrato por el periodo concesional que quedara en el contrato original y compensará al concesionario inicial con los ingresos que se obtengan en la segunda licitación (valor de mercado del negocio).
Por otro lado, el compromiso de no modificación unilateral del contrato por parte de la Administración se ha materializado tradicionalmente, en la legislación española, con el compromiso de reequilibrio económico del concesionario en caso de modificaciones solicitadas por la Administración con posterioridad a la adjudicación del contrato. Este compromiso sigue encontrándose en el artículo 290 de la Ley 9/2017.

La experiencia española muestra que el modelo concesional permitió atraer capital privado a la provisión de infraestructuras. Se logró movilizar recursos adicionales cuando fue necesario cerrar la brecha entre el coste de las infraestructuras y las limitaciones de recursos del sector público. La participación privada ha sido clave a la hora de desarrollar un nuevo modelo de movilidad sostenible, reduciendo los costes de transporte, fortaleciendo la competitividad, el crecimiento económico y la creación de empleo, y mejorando la cohesión territorial.

Esta experiencia ha permitido a las compañías españolas obtener contratos internacionales de concesión. La experiencia doméstica se ha exportado. Así lo ha reconocido en numerosas ocasiones la clasificación de los principales concesionarios a nivel mundial, elaborada por la revista especializada Public Works Financing.

Sin embargo, la experiencia española también adolece de algunas sombras. El éxito de la mayoría de los proyectos facilitó cierto exceso de inversión y un exceso de optimismo a la hora de analizar la viabilidad de determinados proyectos. En particular, ocho concesiones que se adjudicaron entre 1999 y 2004 para la gestión de nueve autopistas de peaje adolecieron de:

- Previsiones de tráfico excesivamente optimistas, que dieron lugar a que las previsiones de ingresos nunca se cumplieran. 
- Previsiones de costes de las expropiaciones demasiado optimistas en un contexto de rápido crecimiento de los precios inmobiliarios, lo que hizo que hubiera sobrecostes de construcción por los mayores costes de expropiación.

El primer factor hizo que, incluso antes de la crisis, ya se estuvieran incumpliendo las previsiones de tráfico de esos proyectos. El advenimiento de la crisis acentuó ese incumplimiento de las previsiones iniciales de tráfico en todas esas autopistas, como se puede ver en el Cuadro 1.

CUADRO 1

DESVIACIONES DE TRÁFICO RESPECTO A LAS PREVISTAS EN LAS NUEVE AUTOPISTAS DE PEAJE EN LIQUIDACIÓN

\begin{tabular}{|l|c|c|}
\hline & 2007 (\%) & 2009 (\%) \\
\hline R-2 Madrid-Guadalajara & -43 & -54 \\
R-3 Madrid-Arganda & -44 & -59 \\
R-5 Madrid-Navalcarnero & -43 & -50 \\
R-4 Madrid-Ocaña & -36 & -56 \\
Eje Aeropuerto & -61 & -71 \\
Ocaña-La Roda & -55 & -56 \\
Madrid-Toledo & -74 & -83 \\
Cartagena-Vera & -70 & N.D. \\
Circunvalación de Alicante & -40 & -55 \\
\hline Fuente: Baeza Muñoz, Ortega Hortelano y Vassallo Magro (sin fecha), \\
página 14.
\end{tabular}

Además, la intensidad media diaria (IMD) de tráfico de estas autopistas siguió cayendo hasta 2013 o 2014, como se puede observar en el Cuadro 2.

En ese mismo cuadro se observa que, a pesar de la recuperación de los últimos años, la mayor parte de estas autopistas de peaje todavía se encuentran lejos de sus niveles de tráfico de $2007^{2}$.

Como consecuencia de todo ello, todas estas concesiones empezaron a padecer problemas financieros y terminaron entrando primero en concurso de acreedores y, entre 2018 y 2019, en fase de liquidación.

Estos casos ponen de manifiesto un diseño inadecuado de las concesiones, con una planificación y evaluación insuficiente de los proyectos.

Existía un reparto de riesgos asimétrico entre el sector público y el privado, ya que, en caso de cancelación anticipada del contrato, el concesionario tenía derecho a recibir la $\triangleright$

2 Las dos excepciones son las autopistas Eje Aeropuerto y CartagenaVera, que fueron aquellas cuyo tráfico cayó menos durante la crisis pero eran la tercera y la segunda, respectivamente, que en mayor medida incumplían ya en 2007 sus previsiones de tráfico iniciales.

CUADRO 2

CAÍDAS MÁXIMAS DEL TRÁFICO EN LAS NUEVE AUTOPISTAS DE PEAJE EN LIQUIDACIÓN Y RECUPERACIÓN HASTA 2017

\begin{tabular}{|c|c|c|c|}
\hline & $\begin{array}{l}\text { Caída máxima acumulada } \\
\text { de la IMD desde } 2007 \text { (\%) }\end{array}$ & Año de caída máxima & $\begin{array}{l}\text { Variación IMD } \\
2007-2017(\%)\end{array}$ \\
\hline R-2 Madrid-Guadalajara & 60,8 & 2014 & $-52,9$ \\
\hline R-3 Madrid-Arganda & 42,5 & 2014 & $-32,4$ \\
\hline R-5 Madrid-Navalcarnero & 42,6 & 2014 & $-37,2$ \\
\hline R-4 Madrid-Ocaña & 60,3 & 2014 & $-52,1$ \\
\hline Eje Aeropuerto & 11,9 & 2013 & 4,3 \\
\hline Ocaña-La Roda & 41,4 & 2013 & $-30,5$ \\
\hline Madrid-Toledo & 51,1 & 2014 & $-33,4$ \\
\hline Cartagena-Vera & 13,9 & 2013 & 12,1 \\
\hline Circunvalación de Alicante ${ }^{1}$ & 47,0 & 2013 & $-35,2$ \\
\hline \multicolumn{4}{|c|}{${ }^{1}$ Primer dato de IMD 2008.} \\
\hline \multicolumn{4}{|c|}{$\begin{array}{l}\text { Fuente: elaboración propia a partir de las tasas de variación anuales (se considera que estos datos son más fiables que los de IMD de cada año, } \\
\text { de la misma fuente, ya que estos datos pueden ser menos homogéneos por la incorporación de nuevos tramos) del Informe } 2017 \text { sobre el } \\
\text { sector de autopistas de peaje en España (páginas } 27 \text { y 28), excepto la tasa de variación entre } 2007 \text { y } 2008 \text { para la autopista Cartagena-Vera, para } \\
\text { la que se ha tomado la IMD de cada año de ese informe, dado que este no da el dato de tasa de variación para ese año. }\end{array}$} \\
\hline
\end{tabular}


RPA, incluso cuando la cancelación era imputable a este (antes de la actual Ley 9/2017). Este aseguramiento de los riesgos soportados por el sector privado pudo animar su participación en un exceso de inversión en infraestructuras y en la incursión en costes ineficientemente elevados en la provisión de las mismas.

Esta red sobredimensionada de infraestructuras y el compromiso de abonar la RPA ha creado pasivos contingentes que, en el caso de las ocho concesiones mencionadas, han cristalizado entre 2018 y 2019.

Ante esta desigual experiencia, resulta conveniente enumerar las principales ventajas, inconvenientes y riesgos de las modalidades contractuales público-privadas.

\section{Ventajas, inconvenientes y riesgos de las PPP}

\subsection{Ventajas}

Las principales ventajas serían:

Menores costes de transacción en comparación con los contratos privados. Frente a los contratos privados, elimina las dificultades de negociar con un elevado número de consumidores. Al mismo tiempo, mantiene del contrato privado la aspiración a describir de manera completa y por anticipado las obligaciones de las partes (Gómez-lbáñez, 2006).

Reducción de riesgos para la Administración. La lógica de utilizar este tipo de estructuras en lugar de la contratación tradicional es que un reparto óptimo de riesgos entre el sector público y el privado genera una mejor relación calidad-precio para la Administración y el usuario (EIB-EPEC, 2019).
Seguridad jurídica para el sector privado. Los contratos de concesión fueron atractivos en los países en desarrollo porque suponían un mayor compromiso por parte de los Gobiernos anfitriones en comparación con la de un regulador discrecional. Para mayor seguridad, estos contratos solían incluir cláusulas de resolución de disputas en tribunales extranjeros o cortes de arbitraje internacionales (Gómez-lbáñez, 2006).

De liquidez. Cuando son servicios rentables, la licitación de los mismos puede permitir a la Administración adelantar ingresos gracias al importe que el adjudicatario esté dispuesto a pagar como precio del contrato.

Adelanto de la disponibilidad de las infraestructuras. La financiación privada permite acometer un mayor número de proyectos que los que serían posibles si se financiaran con cargo a los Presupuestos Generales del Estado, como se ha visto en el caso español.

Equidad intergeneracional. Permite repartir el coste de las infraestructuras entre las generaciones que van a disfrutar de ellas.

Ganancias de eficiencia. La gestión, innovación y know-how privados pueden aportar beneficios para la sociedad que, en cualquier modo, deberán valorarse caso por caso. Además, los procedimientos de adjudicación competitivos bien diseñados permiten maximizar las ganancias de eficiencia. La influencia de las fuerzas del mercado es significativa, aunque no sea tan fuerte como en el caso de los contratos privados, dado que los consumidores no participan directamente, sino que están representados por la Administración Pública (Gómez-lbáñez, 2006).

Garantiza unos niveles de calidad del servicio durante la vigencia del contrato, al tratarse de una obligación contractual. Con ello, se evita que el mantenimiento y la calidad del $\triangleright$ 
servicio dependan de la coyuntura presupuestaria.

Exportaciones de servicios y repatriación de beneficios. Como se ha visto, el desarrollo del sector concesional ha permitido exportar la experiencia.

\subsection{Inconvenientes}

Entre los principales inconvenientes, se pueden citar los siguientes:

Falta de ahorro presupuestario y mayor coste financiero. En el caso de los peajes en sombra y otras modalidades de los PPP con cargo al presupuesto público, lo que se logra es simplemente un diferimiento del coste presupuestario de las infraestructuras, en comparación con la opción de un contrato de obras. Además, el coste de financiación vía presupuestos sería inferior, ya que será el coste de la deuda pública a largo plazo, mientras que el coste de financiación de las concesiones será el coste medio ponderado del capital aportado por el sector privado, que casi siempre será superior. La Comisión Parlamentaria del Tesoro británico llegó a afirmar en 2011 que «la utilización de PFI — siglas habitualmente utilizadas en Reino Unido para PPP - tiene el efecto de incrementar el coste de financiación para las inversiones públicas en comparación con lo que habría estado a disposición del Gobierno si se hubiera endeudado directamente» (Treasury Select Committee, 2011).

Falta de adaptación a las restricciones presupuestarias futuras. Las obligaciones contractuales asumidas por la Administración pueden suponer una carga importante cuando empeora la coyuntura presupuestaria, pudiendo incluso afectar a la calidad de prestación de los servicios públicos. En casos como el de la comisaría de Weymouth East de Dorset, la Policía local tuvo que seguir pagando 2,63 millones de libras al año, a pesar de tener vacío el edificio, por no poder permitirse el coste de su funcionamiento; y en Belfast, un colegio PPP cerró después de siete años, pero el promotor tenía derecho a seguir cobrando 462.500 dólares anuales durante dieciséis años. Además, las onerosas condiciones de pago aceptadas por la Administración británica han generado una presión sobre los presupuestos públicos que ha afectado a otras áreas del mismo. Por ejemplo, el elevado coste del servicio de urgencias del hospital PPP de Calderdale Royal ha obligado a cerrar el servicio de urgencias del hospital público de Huddersfield (Jubilee Debt Campaign, 2017).

Mayor complejidad, que genera costes de consultoría contable y legal (Jubilee Debt Campaign, 2017). Los costes de transacción de los PPP superan ampliamente el $10 \%$ del valor de la inversión en capital (Dudkin y Välilä, 2005).

Erosión de capacidad de la Administración para realizar inversiones. Existe riesgo de que la Administración pierda capacidad para diseñar, construir, financiar y operar infraestructuras internamente (Jubilee Debt Campaign, 2017).

Menor capacidad de participación pública. Por la compleja estructuración de los proyectos bajo esta modalidad contractual y por la confidencialidad de la información societaria que, en la práctica, hace imposible comparar los resultados de los proyectos público-privados con los de los puramente públicos (Jubilee Debt Campaign, 2017).

\subsection{Riesgos}

La experiencia parece indicar que es necesario prestar especial atención a los siguientes factores de riesgo: 
Exceso de optimismo. La Administración debe interpretar las preferencias de los consumidores a la hora de contratar los servicios de infraestructuras (Gómez-lbáñez, 2006) ${ }^{3}$. Al hacerlo, existe un importante riesgo de exceso optimismo porque la mayoría de las ventajas se materializan a corto plazo, a diferencia de los inconvenientes y riesgos, lo que puede dar lugar a errores graves en la asignación de los recursos de la sociedad (caso de las ocho concesionarias de autopistas en proceso de liquidación).

Problemas de equidad intergeneracional. Dado que las infraestructuras son un activo de larga duración, puede tener sentido que todas las generaciones de ciudadanos que vayan a poder beneficiarse de las infraestructuras deban contribuir a su financiación, y no solo aqueIla que contribuía a los ingresos públicos en el momento de la adjudicación de la obra. No obstante, si la infraestructura se financia mediante un bono bullet, el coste de la misma lo soportaría íntegramente la generación de contribuyentes del momento del vencimiento del bono, lo cual también plantea problemas de equidad intergeneracional (Turró y Penyalver, 2019). Además, la pretendida equidad intergeneracional solo puede lograrse si el diseño del proyecto se adapta a las necesidades reales de la sociedad. Si no ${ }^{4}$, varias generaciones pueden terminar pagando por los errores cometidos por la que inició el proyecto, perjudicando, en lugar de favoreciendo, la equidad intergeneracional. Aunque este problema se produce con cualquier modalidad contractual, dados los mayores costes de financiación y de

\footnotetext{
3 Esta desventaja sería común a cualquier participación pública en la provisión de infraestructuras.

4 Por ejemplo, si las necesidades de capacidad están significativamente sobreestimadas y, por ejemplo, la capacidad de una depuradora sobrepasa ampliamente los flujos de aguas residuales que llegan a la misma, o si la IMD de una autopista de peaje está muy por debajo de las previsiones iniciales.
}

estructuración de los PPP, el problema es mayor cuando se utiliza esta modalidad contractual. Por ello, aunque siempre sea muy importante diseñar con precisión una obra, lo es todavía más cuando la modalidad contractual va a ser un PPP.

Riesgo de contratos incompletos. El mayor riesgo y el más frecuente es, al igual que en el caso de los contratos privados, el de que los contratos no contemplen todas las contingencias posibles, sean incompletos y tengan que ser renegociados, lo cual es mucho más complejo que en el caso de los contratos privados, dado que implica un problema de confianza en la Administración, que negocia en nombre de los usuarios. Por ello, ante una situación no prevista por el contrato, el hecho de que cualquier renegociación se hace sin la salvaguardia de una relicitación pone en riesgo la credibilidad de la Administración y puede generar problemas de riesgo moral y oportunismo en las ofertas de los participantes en las licitaciones, todo lo cual pone en peligro el apoyo social a la provisión privada de infraestructuras. En la ola de concesiones de finales del siglo $\mathrm{xx}$, parece que pudo haber presiones que llevaron a concesionar proyectos donde el riesgo de tener contratos incompletos era elevado. Entre las vías para superar estas limitaciones están la inclusión de cláusulas que faciliten la renegociación cuando sea necesaria, o la celebración de contratos de menor duración y simplificados. Este tipo de contratos parece estar detrás del resurgimiento de las concesiones municipales que se produjo en Norteamérica a finales del siglo xx. En ocasiones, se pueden simplificar los contratos dividiéndolos por actividades, separando los contratos de mantenimiento de los de inversión. Sin embargo, esta segmentación también puede, con frecuencia, complicar la supervisión integral de los $\triangleright$ 
contratos (Gómez-lbáñez, 2006). Esta filosofía de reducir el periodo de concesión es la que subyace en la Directiva 2014/23/UE del Parlamento Europeo y del Consejo, de 26 de febrero, relativa a los contratos de concesión, que, con carácter general, limita el periodo de los mismos a cinco años o al mínimo necesario para recuperar las inversiones incluidas en el contrato. Estas mismas condiciones han sido transpuestas en nuestra Ley 9/2017.

Riesgo de inseguridad jurídica. Las partes -especialmente el concesionario- son vulnerables a la profesionalidad e integridad de los tribunales mercantiles o las cortes arbitrales acordadas en los contratos (Gómez-lbáñez, 2006).

Riesgo moral y oportunismo en las licitaciones. Ambas partes tienen incentivo a trasladar parte de sus riesgos a la otra, una vez que el contrato ha sido firmado, si existe cualquier ambigüedad en el mismo.

Riesgo de pasivos contingentes para la Administración. Como pone de manifiesto, entre otros, el caso español de las ocho sociedades concesionarias de autopistas en liquidación, que han devengado una Responsabilidad $\mathrm{Pa}$ trimonial de la Administración (RPA). Incluso un working paper entre dos personas del Departamento Fiscal del FMI reconoce que «han surgido costes y riesgos fiscales elevados derivados de los PPP tanto en países en desarrollo como avanzados (...) los sesgos de los Gobiernos y la manipulación de los PPP añaden una capa importante a los riesgos de los proyectos. Un tratamiento presupuestario y/o estadístico inadecuado puede permitir a los Gobiernos ignorar el impacto de los PPP en el déficit y la deuda públicos. En la práctica, los Gobiernos suelen terminar soportando mayores costes y riesgos fiscales en el medio y largo plazo de los inicialmente previstos» (Jin y Rial, 2016).

\section{Conclusiones}

La experiencia española demuestra que los PPP no siempre constituirán la mejor opción para los contribuyentes. Quizá, los contratos de concesión constituirán la opción más recomendable cuando, bien por la complejidad de los proyectos o su carácter innovador, las ganancias de una gestión integrada de la construcción y posterior explotación y mantenimiento por parte del sector privado —en los casos en los que se produzca- y el valor económico de los riesgos que este asume son mayores que los mayores costes de financiación del sector privado con respecto al público y que los mayores costes de estructuración de esta modalidad contractual. Las ganancias de eficiencia procedentes del sector privado no deben presuponerse y deberían estudiarse caso por caso. Por ejemplo, la Comisión Parlamentaria Selecta del Tesoro británica afirmó en 2011 que no había encontrado evidencia de ahorros y eficiencias durante la vida de los PPP que pudieran compensar los significativamente mayores costes de financiación (Treasury Select Committee, 2011).

Por otro lado, el argumento a favor de adelantar la disponibilidad de las infraestructuras con respecto al momento en que se soporta su coste podría ser relevante en países en desarrollo que se enfrenten a cuellos de botella para su crecimiento o desarrollo económicos por la falta de infraestructuras, pero parece menos sólido en países con una amplia red de infraestructuras, si bien podría haber excepciones.

En todo caso, la conveniencia o no de acudir a este tipo de modalidad contractual dependerá de muchos factores. En un país como España, en algunos sectores como el agua, o la gestión de residuos, las soluciones óptimas $\triangleright$ 
podrían ser considerablemente distintas en unas comunidades autónomas y otras, ya que sus enormes diferencias pueden hacer que un modelo de PPP sea viable en unas regiones pero no en otras, o que lo sea solo si se adoptan medidas particulares.

Por ello, es necesario que las autoridades contratantes tengan un mejor conocimiento, para cada sector, sobre en qué casos es preferible acudir a las figuras tradicionales de los contratos de obra o de servicios. La decisión debería basarse en una comparación del coste para los contribuyentes de ambas opciones, con un enfoque de largo plazo y sin subestimar posibles pasivos contingentes.

A nivel internacional, este ejercicio se realiza mediante una herramienta que, en inglés, recibe la denominación de value for money. Sin embargo, en la legislación española no existe ninguna obligación de emplearla, lo que acentúa el riesgo de optar por la modalidad concesional en proyectos en los cuales los contratos públicos tradicionales podrían salir más rentables a largo plazo a los contribuyentes.

Además, existe siempre el riesgo de que, una vez firmado el contrato, ambas partes tengan incentivos al tratar de aprovechar cualquier ambigüedad en el mismo para transferir riesgos de vuelta a la otra.

La necesidad de evitar el exceso de optimismo, de garantizar la viabilidad de los proyectos que se proponen en modalidad PPP y una reducción efectiva de los riesgos soportados por los contribuyentes llevó a que, por medio del artículo 333 de la Ley $9 / 2017^{5}$, se creara la Oficina Nacional de Evaluación, que tiene como finalidad analizar la sostenibilidad financiera de los contratos de concesión y si la rentabilidad

5 En realidad, formalmente, ya se creó por medio de la disposición final 9. ${ }^{\text {a }}$ doce de la Ley 40/2015, de 2 de octubre, de Régimen Jurídico del Sector Público, pero no había llegado a ponerse en funcionamiento. del proyecto es razonable en atención al riesgo de demanda que asume el concesionario.

El informe de la Oficina Nacional de Evaluación es preceptivo cuando la Administración realiza cualquier tipo de apoyo financiero al concesionario, como, por ejemplo, un pago por disponibilidad o un peaje sombra.

Por lo tanto, si se dota a la Oficina Nacional de Evaluación de suficientes recursos, tanto desde el punto de vista cuantitativo como desde el punto de vista cualitativo, y se convierte en una verdadera unidad de PPP de referencia para España, puede aportar un enorme valor añadido a las autoridades contratantes españolas a la hora de saber cuándo la modalidad de concesión aporta valor a largo plazo a los contribuyentes y para evitar repetir errores del pasado.

Por otro lado, hay que alertar sobre el hecho de que el mandato de la oficina, tal como está actualmente recogido en la Ley de Contratos, no evitaría necesariamente la repetición de algunos de los mayores errores del pasado, como el de las ocho concesiones de autopistas fallidas. En efecto, puesto que se suponía que esas autopistas se financiarían exclusivamente con cargo a los usuarios y no iban a necesitar ningún apoyo financiero público, el informe de la Oficina Nacional de Evaluación, de haber existido entonces, no habría sido preceptivo en esos casos y no lo sería si alguien propusiera proyectos similares en el futuro. Por ello, para evitar estas situaciones, sería recomendable reformular, en el artículo 333.3, los casos en los que el informe es preceptivo.

En resumen, la satisfacción de las necesidades de la población por medio de servicios públicos de calidad requiere un grado importante de inversiones y mantenimiento para lograr que sean sostenibles. El sector privado podría desempeñar un papel importante en $\triangleright$ 
la satisfacción de estas necesidades, pero su potencial debería evaluarse con cuidado para evitar que sea sobreestimado. Para ello, la Oficina Nacional de Evaluación deberá convertirse en una verdadera unidad PPP de España, dotada con los recursos apropiados y centrada en aquellos proyectos con mayor riesgo de convertirse en una carga para los presupuestos o de esconder importantes pasivos contingentes, con independencia de si se financian vía presupuestos o con cargo a los usuarios.

\section{Bibliografía}

Baeza Muñoz, M.Á., Ortega Hortelano, A., y Vassallo Magro, J.M. (2011). La crisis económica en las concesiones de autopistas de peaje. Recuperado de http://oa.upm.es/11837/1/INVE_ MEM_2011_103800.pdf

Cañas Fuentes, M., Sánchez Soliño, A., Vassallo Magro, J.M., y Castroman Pollero, Á. (2006). Modernización y conservación de carreteras mediante peaje sombra. Universidad Politécnica de Madrid. Recuperado de http://www2.caminos.upm.es/Departamentos/construccion/Economia/catedra/ doc/FOMENTO\%20PEAJE\%20SOMBRA.pdf

Delegación del Gobierno en las Sociedades Concesionarias de Autopistas Nacionales de Peaje, Ministerio de Fomento (2018). Informe 2017 sobre el sector de autopistas de peaje en España. Madrid, España. Recuperado de https://apps. fomento.gob.es/CVP/handlers/pdfhandler. ashx?idpub=ICW039

Department of Housing, Planning and Local Government -Ireland- (2006). Assessment of Projects for Procurement as Public Private Partnerships. Central Guidance Note No. 6 (Technical) November. Public Private Partnership/Comhpháirtíocht Phoblí Phríamháidrach. Irlanda.

Dudkin, G., y Välilä, T. (2005). Transaction costs in public-private partnerships: a first look at the evidence. Economic and Financial Report 2005/03,
European Investment Bank. Recuperado de https://www.eib.org/attachments/efs/efr_2005_ v03_en.pdf

European Investment Bank (2019). EPEC European PPP Expertise Centre. How can I help you? Frequently asked questions. Luxemburgo: EIB. Recuperado de https://www.eib.org/epec/find-outmore/faq.htm

Gómez-lbáñez, J.A. (2006). Regulating infrastructure. Monopoly, contracts, and discretion. Cambridge, Massachusetts, Estados Unidos, y Londres, Reino Unido: Harvard University Press.

Jin, H., y Rial, I. (2016). IMF Fiscal Affairs Department, Regulating Local Government Financing Vehicles and Public-Private Partnerships in China. Recuperado de http://www.imf.org/external/ pubs/ft/wp/2016/wp16187.pdf

Jubilee Debt Campaign (2017). The UK's PPPs Disaster. Lessons on private finance for the rest of the World. Londres, Reino Unido. Recuperado de https://jubileedebt.org.uk/wp-content/ uploads/2017/02/The-UKs-PPPs-disaster_Final-version_02.17.pdf

Ley 8/1972, de 10 de mayo, de Construcción, Conservación y Explotación de Autopistas en Régimen de Concesión. Boletín Oficial del Estado, núm. 113, de 11 de mayo de 1972, pp. 8229 a 8234. Recuperado de https://www.boe.es/boe/ dias/1972/05/11/pdfs/A08229-08234.pdf

Ley 55/1999, de 29 de diciembre, de Medidas Fiscales, Administrativas y del Orden Social. Boletín Oficial del Estado, núm. 312, de 30 de diciembre de 1999, pp. 46095 a 46149. Recuperado de https://www.boe.es/boe/dias/1999/12/30/pdfs/ A46095-46149.pdf

Ley 9/2017, de 8 de noviembre, de Contratos del Sector Público, por la que se transponen al ordenamiento jurídico español las Directivas del Parlamento Europeo y del Consejo

2014/23/UE y 2014/24/UE, de 26 de febrero de 2014. Boletín Oficial del Estado, núm. 272, de 9 de noviembre de 2017, pp. 107714 a 108007 . $\triangleright$ 
Recuperado de https://www.boe.es/boe/dias/ 2017/11/09/pdfs/BOE-A-2017-12902.pdf

Treasury Select Committee, de la Cámara de los Comunes del Reino Unido de Gran Bretaña e Irlanda del Norte (2011, 19 de agosto). Treasury - Seventeenth Report. Private Finance Initiative. Londres, Reino Unido. Recuperado de https://publications. parliament.uk/pa/cm201012/cmselect/cmtreasy/ 1146/114608.htm y de https://www.parliament.uk/ business/committees/committees-a-z/commons-select/treasury-committee/news/pfi-report/

Turró, M., y Penyalver, D. (2019). Hunting white elephants on the road. A practical procedure to detect harmful projects of transport infrastructure. Research in Transportation Economics, Elsevier, 75, 3-20. Recuperado de https://www.sciencedirect.com/journal/research-in-transportationeconomics/vol/75/suppl/C 